\title{
Hormone replacement therapy and the prevention of postmenopausal osteoporosis
}

\author{
Marco Gambacciani ${ }^{1}$, Marco Levancini ${ }^{1,2}$ \\ ${ }^{1}$ Department of Obstetrics and Gynecology, Pisa University Hospital, Italy \\ ${ }^{2}$ Department of Obstetrics and Gynecology, Clinica Alemana, Univesidad Del Desarrollo, Santiago, Chile
}

\begin{abstract}
Fracture prevention is one of the public health priorities worldwide. Estrogen deficiency is the major factor in the pathogenesis of postmenopausal osteoporosis, the most common metabolic bone disease. Different effective treatments for osteoporosis are available. Hormone replacement therapy (HRT) at different doses rapidly normalizes turnover, preserves bone mineral density (BMD) at all skeletal sites, leading to a significant, reduction in vertebral and non-vertebral fractures. Tibolone, a selective tissue estrogenic activity regulator (STEAR), is effective in the treatment of vasomotor symptoms, vaginal atrophy and prevention/treatment of osteoporosis with a clinical efficacy similar to that of conventional HRT. Selective estrogen receptor modulators (SERMs) such as raloxifene and bazedoxifene reduce turnover and maintain or increase vertebral and femoral BMD and reduce the risk of osteoporotic fractures. The combination of bazedoxifene and conjugated estrogens, defined as tissue selective estrogen complex (TSEC), is able to reduce climacteric symptoms, reduce bone turnover and preserve BMD. In conclusion, osteoporosis prevention can actually be considered as a major additional benefit in climacteric women who use HRT for treatment of climacteric symptoms. The use of a standard dose of HRT for osteoporosis prevention is based on biology, epidemiology, animal and preclinical data, observational studies and randomized, clinical trials. The antifracture effect of a lower dose HRT or TSEC is supported by the data on BMD and turnover, with compelling scientific evidence.
\end{abstract}

Key words: menopause, osteoporosis, estrogen, prevention, fracture.

\section{Introduction}

Fracture prevention is considered one of the public health priorities by the World Health Organization [1]. Osteoporosis is a major healthcare problem leading to a high incidence of spine, radial, and mainly hip fractures that are causes of morbidity and mortality in ageing population [2-4]. It is recognized as a systemic skeletal condition characterized by low bone mass, microarchitectural deterioration of bone tissue, and compromised bone strength leading to enhanced bone fragility and a consequent increased risk of fractures. Bone strength reflects the integration of two main features: bone density and bone quality [1]. Osteoporosis affects mostly postmenopausal women: $30-50 \%$ of women will suffer a clinical fracture and the associated morbidity in the course of their lifetime and $70 \%$ of hip fractures occur in women [2]. Postmenopausal osteoporosis is estimated to affect 200 million women worldwide, 75 million in Europe, the USA and Japan alone [2]. Osteoporotic fractures not only represent a big economic cost, but also lead to long-term consequences like chronic pain, deformity, depression, disability and death with a large burden of non-hip fractures being underestimated [3]. Hip fracture is responsible for a large proportion of the financial burden of osteoporosis to health-care systems, but other osteoporosis-related fractures, particularly vertebral fractures, cause considerable morbidity. Vertebral fractures are often not diagnosed and not treated, although they are common osteoporosis fractures: a 50-year-old woman has a 16\% lifetime risk of experiencing a vertebral fracture and it is estimated that only about a fifth to half of them are diagnosed and treated [3]. There are various effective drug treatments for the prevention and treatment of osteoporosis. Today osteoporosis is under-diagnosed and under-treated notwithstanding the fact that effective prevention and treatment options are available. Osteoporosis is a global problem that will further increase over the next 50 years. Measures are urgently required to avert this trend. Although important organizations are issuing guidelines and recommendations, increased awareness of the burden of the disease is required. Other critical problems are the access to diagnostic technologies, which is limited by both availability and funding, and the availability of reimbursement for ap- 
propriate treatments. In this review we summarize the evidence for the hormone use in postmenopausal years for the prevention of postmenopausal osteoporosis.

\section{Diagnosis}

Diagnosis is based on bone mineral density (BMD) assessment by dual X-ray absorptiometry (DXA), expressed as the $T$-score, and/or the presence of fragility fractures (Table I). The World Health Organization (WHO) defines a fragility fracture as "a fracture caused by injury that would be insufficient to fracture a normal bone: the result of reduced compressive and/ortorsional strength of the bone". Therefore, clinically, a fragility fracture may be defined as one that occurs as a result of minimal trauma, such as a fall from a standing height or less, or no identifiable trauma. Low bone mass is a risk factor for fractures at nearly all skeletal sites [4]. However, it has been claimed that BMD assessment is not a cost-effective population screening tool and is best applied based on age and other risk factors [5]. Worldwide the osteoporotic and normal patients are defined according to the "T-score", which is the number of standard deviations above or below the mean BMD for normal young adults as follows:

1) normal BMD is defined as a $T$-score between +2.5 and -1.0 (i.e., the patient's BMD is between 2.5 standard deviations (SDs) above the young adult mean and one SD below the young adult mean);

2) osteopenia (low BMD) is associated with a T-score between -1.0 and -2.5 , inclusive;

3) osteoporosis is defined as a $T$-score lower than -2.5;

4) severe or established osteoporosis defines patients whose $T$-score is below -2.5 and who also have suffered a fragility fracture.

The probability of fracture in an individual can be estimated using different models and algorithms that integrate various risk factors for fracture. They include the FRAX ${ }^{\oplus}$ model developed by the World Health Organization [5]. FRAX ${ }^{\circledast}$ estimates the 10-year probability of fracture combining clinical risk factors (Table II) with or without BMD (available online at www.sheffield.ac.uk/ $F R A X /$ ). FRAX ${ }^{\circledast}$ was intended to support treatment decisions, optimizing the diagnostic and treatment resourc-

Tab. I. WHO definition for osteoporosis. The $T$-score compares an individual's BMD with the mean value for young normal individuals and expresses the difference as a standard deviation score

\begin{tabular}{lc}
\hline Category & $T$-score \\
\hline Normal & -1.0 and above \\
\hline Low bone mass (osteopenia) & -1.0 to -2.5 \\
\hline Osteoporosis & -2.5 and below \\
\hline
\end{tabular}

es. Thus, FRAX ${ }^{\oplus}$, which is not a diagnostic tool, calculates the 10-year probability for hip, vertebrae, wrist, and proximal humerus fractures. However, we have to underline that FRAX ${ }^{\oplus}$ does not consider the dose and duration of exposure to corticosteroids, tobacco and alcohol, and some risk factors for fractures are not even included in the model (physical activity, falls, vitamin D deficiency, markers of bone turnover, previous osteoporosis treatment, or the use of different drugs such as anticonvulsants, GnRH agonists, aromatase inhibitors, and androgen deprivation). In addition, the FRAX ${ }^{\circledast}$ calculation model does not consider lumbar spine BMD (only the femoral neck), or the presence, number and severity of vertebral fractures. Therefore, FRAX ${ }^{\circledast}$ has intrinsic limitations that may underestimate the individual risk of fractures. An individual, full clinical appropriate assessment of familiar and personal history, prevalent fractures and secondary causes of osteoporosis should precede any therapeutic decisions.

The simple measurement of BMD does not seem to fulfill the required criteria to be offered as a screening. One way to identify high-risk patients is to select those with clinical risk factors and to perform BMD measurement, and if they are under the threshold for osteoporosis ( $-2.5 T$-score), then treat them. This may

Tab. II. Risk factors considered in FRAX ${ }^{\circledR}$

\begin{tabular}{l}
\hline Country of residence \\
\hline Race (only for the U.S. model: White, Hispanic, African, \\
American, Asian) \\
\hline Age: accepts ages between 40 and 90 years \\
\hline Gender: male - female \\
\hline Weight $(\mathrm{kg})$ and height $(\mathrm{cm})$ : used to calculate BMI \\
\hline Previous fracture: spontaneous in adult life, or traumatic but \\
would not have occurred in a healthy individual \\
\hline Family history: parent with hip fracture
\end{tabular}

Corticosteroids: prednisone $5 \mathrm{mg} /$ day for 3 months in the past or present

Rheumatoid arthritis (diagnosis confirmed)

\begin{tabular}{l}
\hline Smoking (current) \\
\hline Alcohol: 3 drinks per day \\
\hline Secondary osteoporosis \\
\hline Diabetes mellitus type I \\
\hline Osteogenesis imperfecta in adults \\
\hline Long-standing untreated hyperparathyroidism \\
\hline Hypogonadism or premature menopause $(<45$ years) \\
\hline Chronic malnutrition or intestinal malabsorption \\
\hline Chronic liver disease \\
\hline BMD: $T$-score or g/cm ${ }^{2}$ at the femoral neck
\end{tabular}


be conservative. An alternative is to offer a BMD screen to all the individuals older than 65 if they have a clinical risk of fractures. The BMD threshold for treatment could be even lower $(-1.5 T$-score if there are other risk factors or, $-2.0 T$-score w/o risk factors). There are other independent risk factors for fractures which should be taken into account in parallel with BMD. Age and prior vertebral fracture are the most important ones. Other relevant risk factors are low body mass index, smoking, steroid use ever, and alcohol use. The combined use of different risk factors could allow for a better stratification of the risk of fractures.

\section{Pathogenesis and clinical management}

Postmenopausal osteoporosis can be caused by the failure to attain peak bone density or accelerated bone loss after menopause (Fig. 1). The attainment of an optimal peak bone mass is important in the prevention of osteoporosis. Optimal skeletal health is dependent on genetics, with an appropriate lifelong balance of diet and lifestyle factors, such as weight-bearing exercise and the avoidance of bone-toxic substances. Bone mass acquisition occurs during childhood, adolescence and early adult life. Low peak bone mass can be determined by genetic factors, inadequate nutrition during growth and development (particularly calcium and protein intake), limited physical activity, concurring diseases (e.g. thyrotoxicosis, Cushing's) or drugs (e.g. corticosteroids, anticonvulsants) during growth, which impair bone mass acquisition. Particularly, in women, low exposure to reproductive hormones, may reduce the bone mineral density, such as hypothalamic amenorrhea patients (Fig. 2). The combination of malnutrition and the hypoestrogenism such as anorexia nervosa patients may cause a further reduction in peak bone mass (Fig. 2). The estrogen deficiency is a key factor in the pathogenesis of osteoporosis in otherwise normal postmenopausal women $[6,7]$. All conditions of estrogen deficiency are associated with a significant bone loss [7]. In healthy normal women, estrogen decline is the major, or the sole, cause of accelerated bone turnover and the consequent decrease in bone density and architectural damage of bone structure, leading to a decreased bone strength that is bound to an increased fragility fracture risk. It appears logical that the restoration of estrogen levels may represent the preventive measure of choice in climacteric women.

Although both men and women experience bone loss as a natural part of the aging process, bone loss progresses rapidly in postmenopausal women $[6,7]$. The goal of management in osteoporosis is the prevention of fractures. Choice of therapy should be based on a balance of effectiveness, risks and costs. Clinical management in osteoporosis can be discussed in terms of prevention and treatment. Prevention in osteoporosis

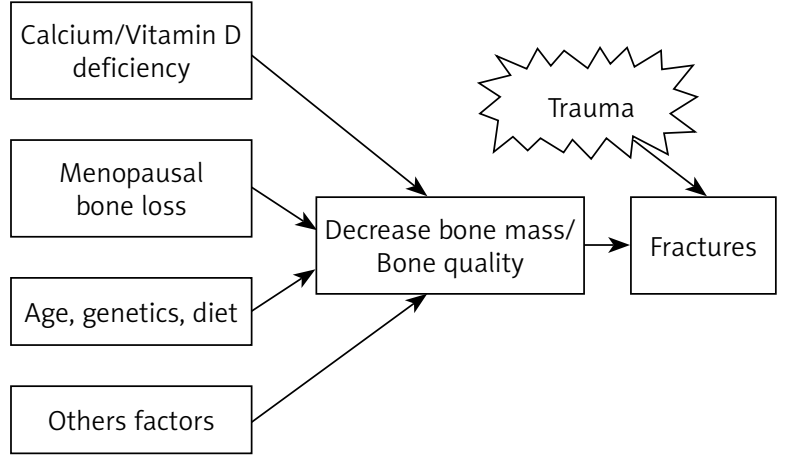

Fig. 1. Pathophysiology of osteoporosis-related fractures

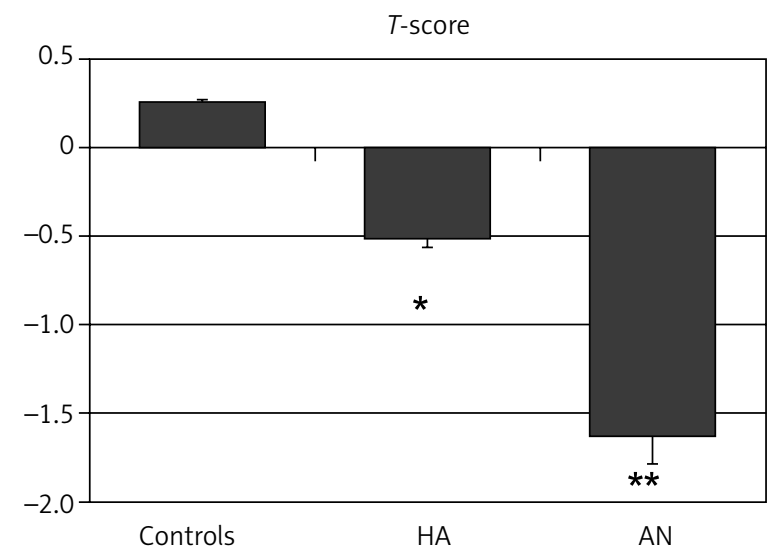

Fig. 2. Osteopenia in young women with hypothalamic amenorrhea (HA) and anorexia nervosa (AN) patients. The figure shows the $T$-scores for lumbar spine BMD measured in normal control women $(n=30)$ and patients with $\mathrm{HA}(n=23)$ and $\mathrm{AN}$ $(n=15) .{ }^{*} p<0.01$ vs. Controls; ${ }^{* *} p<0.001$ vs. Controls and HA

means intervention that creates an environment and basic lifestyle that ensures a high peak bone mass and its preservation. Primary prevention of osteoporosis is directed at women identified as being at an increased risk, but without established disease. Adequate nutrition and exercise are recommended, eliminating risk factors such as alcohol abuse and smoking. In this view, prevention includes the maintenance of a normal and balanced estrogen stimulation on bone throughout the reproductive life. Conversely, treatment consists in intervention in patients with established osteoporosis to reduce the risk of further fractures and to decrease the morbidity associated with the fracture. There is no consensus on the criteria to select the patients to be treated. The decision is driven also by the costs of antiosteoporotic drugs. Accordingly, we have to consider that hormone replacement therapy (HRT) can be defined as an inexpensive osteoporosis treatment, having additional benefits on climacteric symptoms and quality of life. Vasomotor symptoms have been linked to risk factors for midlife women's mental and physical health, as well as lower BMD $[8,9]$. In these symptomatic women, HRT may face not only the issue of symptoms and qual- 
ity of life, but also the issue of osteoporosis prevention. Climacteric symptoms may be a key factor for initiation of HRT in perimenopausal and early postmenopausal women presenting with low BMD or risk factors for osteoporosis.

\section{Osteoporosis and hormone replacement therapy}

Since the major underlying cause of postmenopausal osteoporosis is the loss of bone resulting from estrogen deficiency, HRT is the rational approach in peri- and postmenopausal women [10-20]. However, nowadays HRT is not considered as the first-line treatment for osteoporosis by different Medical Societies and Associations based on the safety concerns raised by the results of Women Health Initiative study (WHI) and Million Women's Study [21-23]. However, these concerns have been largely revised by the International Menopause Society and other Scientific Societies [24, 25]. In the Global Consensus Statement on Menopausal Hormone Therapy (endorsed by the American Society for Reproductive Medicine, the Asia Pacific Menopause Federation, the Endocrine Society, the European Menopause and Andropause Society, the International Menopause Society, the International Osteoporosis Foundation and the North American Menopause Society), it has been clearly stated that HRT is effective and appropriate for the prevention of osteoporosis-related fractures in at-risk women before the age of 60 years or within 10 years after menopause [25].

Therefore, in postmenopausal women at risk of fracture and younger than 60 years, or within 10 years of menopause, HRT can be considered as one of the first-line therapies for the prevention and treatment of osteoporosis-related fractures. Conversely, the initiation of standard HRT after the age of 60 years for the exclu-

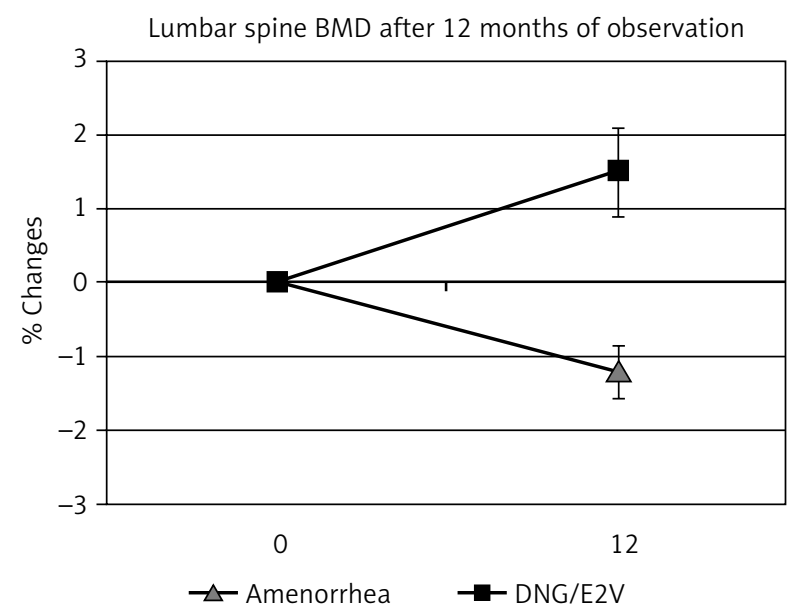

Fig. 3. Effects of combined oral contraceptive containing dienogest/estradiol valerate (DNG/E2V) in functional hypothalamic amenorrhea patients. Mean age $21.5 \mathrm{yr}$.; $n=21$ in each group sive reason for fracture prevention is not recommended since the potential risk of long-term complications, namely breast cancer, can outweigh the benefits [24]. Thus, the extension of HRT after the age of 60 years must take into account potential long-term benefits and risks of the specific dose and route of administration, compared to other proven non-hormonal therapies [24].

The protective effect of HRT on bone mineral density declines after cessation of therapy at an unpredictable rate, although some degree of fracture protection may remain after cessation of HRT [26]. If the patient is still considered at risk for fracture after cessation of HRT, additional therapy with proven bone-sparing medication should be given.

Hormone replacement therapy is able to preserve and even increase BMD at all skeletal sites, such as lumbar spine, femoral neck and forearm in postmenopausal women [13]. Likewise, the administration of a low dose oral contraceptives can reverse the deleterious effect of hypoestrogenism in young women (Fig. 3). In premenopausal women with oligomenorrhea or amenorrhea characterized by low estrogen production, the administration of low dose oral contraceptives is the treatment that can restore bone turnover and normal bone density jeopardized by an untimely decrease in estrogen production [12] (Fig. 4). The estrogen supplementation can be easily performed with new oral contraceptive formulations, containing natural estrogen. We have recently treated oligo-amenorrheic premenopausal women with the combined oral contraceptive (COC) containing dienogest/estradiol valerate (DNG/E2V). We evaluated the effects on BMD (Figs. 3 and 4) and on serum and urinary bone turnover markers (data not reported). In DNG/ E2V-treated women, a significant $(p<0.05)$ increase in

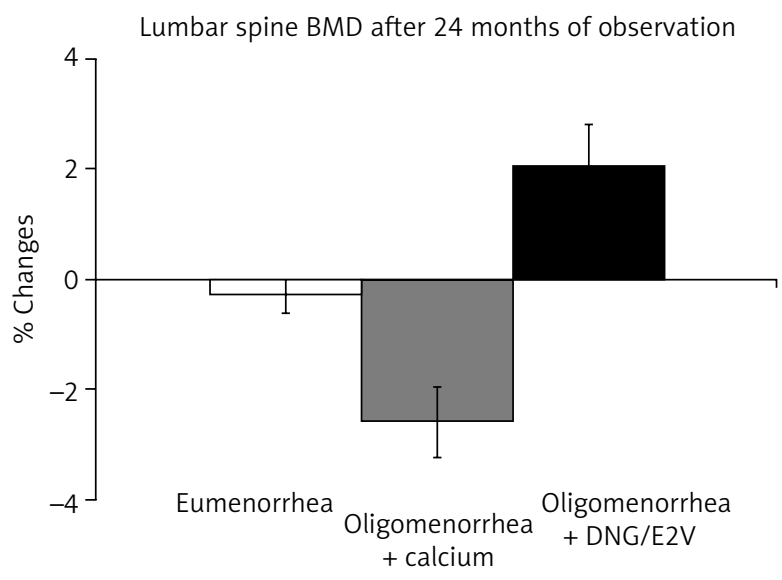

Fig. 4. Effects of combined oral contraceptive containing dienogest/oestradiol valerate (DNG/E2V) in perimenopausal women. The figure reports the percent variation of bone mineral density (BMD) measured by DXA (Lunar Corporation) in eumenorrhoic, oligomenorrhoic (supplemented with $500 \mathrm{mg}$ calcium a day) and oligomenorrhoic-OC treated perimenopausal women (mean age $49.5 \pm 2.1 \mathrm{yr}$; $n=25$ in each group) 
bone density was observed (Figs. 3 and 4). Therefore, replacement therapy of low dose oral contraceptives in premenopausal women suffering from functional hypothalamic hypoestrogenic amenorrhea (Fig. 3), or in perimenopausal patients can also reverse the effects of hypoestrogenism on BMD [12] (Fig. 4).

The greatest benefits from HRT in terms of bone sparing effects can be obtained shortly after the menopause, although HRT prevents bone loss in all stages of postmenopausal life, at least up to an age of 70 years [13].

In conclusion, since estrogen decline is the major cause of osteoporosis in women, and estrogen administration is effective in preventing the climacteric consequences on bone density and metabolism, HRT can be seen as an effective option for prevention of osteoporosis in peri- and postmenopausal women.

\section{Hormone replacement therapy and fracture}

Hormone replacement therapy decreases the incidence of all osteoporosis-related fractures, including vertebral and hip fractures, even in women not at a high risk of fracture $[10,19,21,24]$. Findings of epidemiological and observational studies are now supported by clinical randomized trials, demonstrating that HRT is associated with a $30 \%$ significant reduction in vertebral and non-vertebral fractures [21]. A meta-analysis [19], including 22 fracture trials, concluded that HRT significantly reduced non-vertebral fractures $(R R=0.73 ; \mathrm{Cl}$ : 0.56-0.94), but the effect was attenuated and not statistically significant in women older than 60 years. Evidence for protection against of the fracture is limited to standard dosages of conjugated equine estrogen (CEE) and medroxyprogesterone acetate (MPA), given by the oral route. In the $\mathrm{WHI}$ trial, women assigned to active treatment (CEE, $0.625 \mathrm{mg} /$ day, plus MPA, $2.5 \mathrm{mg} /$ day) had fewer fractures compared with women assigned to placebo ( $\mathrm{HR}=0.76 ; \mathrm{Cl}: 0.69-0.83)$ [21]. The effect did not differ in women stratified by BMI, age, time since menopause [21]. The $\mathrm{WHI}$ is the first randomized trial with definitive data supporting the ability of postmenopausal HRT at standard doses to prevent fractures at the hip, vertebrae, and other sites. The WHI findings are particularly relevant since the study subjects were not specifically selected on the basis of a high risk of osteoporosis and related fracture or a known history of os- teoporosis (with or without prior fracture). Thus, standard HRT is effective in preventing bone loss associated with the menopause and decreases the incidence of all osteoporosis-related fractures, including vertebral and hip fractures, even in women not selected for a high fracture risk.

Based on evidence of effectiveness, cost and safety, standard HRT should be considered one of the first-line therapies for the prevention and treatment of fractures in postmenopausal women, younger than 60 years $[24$, 25]. Conversely, according to the available evidence, the initiation after the age of 60 years of HRT for the sole purpose of fracture prevention is not recommended. In women that are already on HRT, the continuation of HRT after the age of 60 years should take into account the possible long-term effects of the specific dose and method of administration of HRT, compared to other proven non-hormonal therapies [24, 25].

\section{Low-dose hormone replacement therapy}

Many women drop out of the standard dose HRT because of the side effects, and bone sparing effect can be lost a few years after discontinuation [26]. The strategy to maintain the beneficial estrogen effect on bone is to improve long-term compliance and continuation reducing the HRT dosage. The usually prescribed HRT dosage has declined progressively over the past 20 years [24, 27-30]. The use of low and ultra low dose of estrogen has grown in popularity (Table III). Various studies have assessed the efficacy of lowdose HRT (LD-HRT) and tibolone in the prevention of osteoporosis in postmenopausal women, showing that LD-HRT can decrease the bone turnover rate by approximately $30 \%$, with an increase in bone density [28, 29]. The HOPE (Health, Osteoporosis, Progestin, Estrogen) trial, a large, randomized placebo-control trial demonstrates that lower doses of CEE ( 0.45 and $0.3 \mathrm{mg} / \mathrm{day})$, with or without a lower dose of MPA (1.5 mg/day), prevented the loss of spine and hip BMD and reduced bone turnover in early postmenopausal women [29]. In addition, the HOPE trial demonstrated the beneficial effects of these low-dose regimens on vasomotor symptoms and vaginal atrophy, lipid profiles, bleeding profiles, and endometrial hyperplasia[29]. Thus, LD-HRT mayenhance patient continuation, with adequate bone protection and menopausal symptom control. LD-HRT gives physicians the possibility to personalize the doses on the ba-

Tab. III. Estrogen and tibolone daily doses as commonly referred

\begin{tabular}{lcccc}
\hline & Oral estradiol (mg) & Conjugated estrogens (mg) & Transdermal estradiol (mcg) & Tibolone (mg) \\
\hline Standard & 2 & 0.625 & 50 & 2.5 \\
\hline Low dose & 1 & 0.45 & 25 & 1.25 \\
\hline Ultra low dose & 0.5 & 0.30 & 12.5 & - \\
\hline
\end{tabular}


sis of each individual patient's needs. In addition, ultralow dose estrogen could be appropriate as a new start for women aged 60 and older, who might benefit from modest increases in estrogenic action sufficient for their age to preserve skeletal integrity, possibly without significant breast effects and endometrial stimulation.

\section{Postmenopausal women and osteoarthritis}

Osteoarthritis (OA) is an aging-related chronic joint disease. The social and economic impact of the disease is tremendous as OA is the major reason for disability and reduced quality of life among older people [31]. Preclinical studies demonstrate that estrogen decline can have a major effect not only in the pathogenesis of osteoporosis but also of OA $[32,33]$. Such an effect is prevented and in some cases reversed with estrogen therapy [33]. The marked predominance of polyarticular osteoarthritis in women and, in particular, the marked increase in osteoarthritis in women after the menopause both point to a likely involvement of female sex steroids in the maintenance of cartilage homeostasis. Estrogen receptors have been identified in the intervertebral disk and estrogen has a protective, mitogenic effect [34]. Apart from its positive effect on the bone, it has been recently found that estrogen induces favorable changes in the intervertebral discs. After menopause, intervertebral disk space shows a progressive decrease that almost entirely occurs in the first 5-10 years since menopause, suggesting that the estrogen decrease may rapidly change connective tissue metabolism in the intervertebral disks [35]. Estrogen decline after menopause leads to changes in the connective tissue matrix and estrogen therapy may prevent and at least in part reverse these changes [36]. These effects are relevant since disc space narrowing is a clear risk factor for vertebral fracture. In addition, the stimulation of estrogen receptor by estrogen and a selective estrogen receptor modulator (SERM) may protect postmenopausal women from an excessive cartilage turnover [37]. In fact, in postmenopausal women treated with SERMs, the urinary excretion of C-telopeptide of type II (CTX-II), a biomarker for cartilage turnover, is decreased by approximately $50 \%$, restoring CTX-II levels into the premenopausal range [28]. Thus, timely initiated estrogen/SERM treatment can effectively prevent both bone and cartilage loss accompanying the menopause, involving both direct and indirect mechanisms, exerting a chondro-protective effect, with a decreased incidence of osteoarthritis, suggesting potential benefits of HRT in the prevention of destructive joint diseases such as osteoarthritis.

\section{Tibolone}

Tibolone is a synthetic steroid, a norethisterone derivative, metabolized to molecules that have affinity for the estrogen, progesterone and androgen receptors, which has been described as a selective estrogenic activity regulator (STEAR). Different tibolone doses resulted in a suppression of bone turnover and long-term positive effects on bone mineral density measured at different skeletal sites [38]. A low dose of $1.25 \mathrm{mg} /$ day was studied at the Long-term Intervention on Fractures with Tibolone (LIFT) [39] resulting in a reduction of vertebral and non-vertebral fracture risk in comparison to placebo. In this study, tibolone was associated with an increased risk of stroke. This deleterious effect on stroke risk must be ascribed to the age of the population treated. In fact, in all studies in which the standard dose of tibolone was used, no increased risk of stroke was reported [24]. In addition, the low-dose tibolone used in the LIFT study was associated in a reduced risk of endometrial, colon and breast cancer, and no increase in the risk of venous thromboembolism was seen [39]. In conclusion, tibolone is effective in the treatment of vasomotor symptoms, vaginal atrophy and prevention/treatment of osteoporosis with a clinical efficacy similar to that of conventional HRT.

\section{Selective estrogen receptor modulators}

The concerns on long-term use of estrogens have focused the attention on strategies to reduce the possible impact of estrogen on the breast cancer risk.

The selective estrogen modulators (SERMs) are chemically different compounds that lack the steroid structure of estrogens, but are able to interact with estrogen receptors as agonists or antagonists depending on the target tissue. The early SERMs, tamoxifen and raloxifene were originally developed for the prevention and treatment of breast cancer and were subsequently found to conserve bone mass. Tamoxifen has been used for over 30 years, either as adjuvant treatment of, and also to prevent breast cancer incidence in high-risk women. Tamoxifen showed a significant bone sparing effect [40], but its use was linked with increased risks of endometrial cancer, stroke, pulmonary emboli, deep-vein thrombosis, and cataracts, and thus it is not indicated for the prevention or treatment of postmenopausal osteoporosis.

Raloxifene is the first SERM approved for the treatment and prevention of osteoporosis in postmenopausal women in the United States and Europe. Raloxifene is used for postmenopausal osteoporosis prevention worldwide, since it was shown to reduce bone turnover and increase BMD, conferring a 30-50\% risk reduction in vertebral, but not non-vertebral fracture [41] and it is as effective as tamoxifen in reducing the risk of invasive breast cancer, with a significantly lower risk of endometrial hyperplasia, thromboembolic events, and cataracts than tamoxifen [42]. 
A third-generation SERM, bazedoxifene was extensively evaluated in preclinical studies producing convincing data supporting its use as an antiresorptive agent for the prevention and treatment of postmenopausal osteoporosis. Bazedoxifene reduces bone turnover and maintains or increases vertebral and femoral BMDs in comparison to placebo [43]. In a 3-year RCT, placeboand active-controlled trial of 7,492 women, bazedoxifene reduced the risk for new vertebral and non-vertebral fractures in high-risk women [44]. Bazedoxifene is safe and well tolerated, with no evidence of endometrial or breast stimulation. These data suggest that bazedoxifene may offer significant clinical benefits for postmenopausal women with or at risk of osteoporosis.

A new goal in preventing postmenopausal osteoporosis is the combination of a SERM with an estrogen, in order to treat climacteric syndrome, preventing the bone consequences of estrogen decline, and in the meantime, avoiding the use of progestogens. The combination of a SERM with an estrogen has been defined as tissue selective estrogen complex (TSEC). This novel approach has been evaluated with bazedoxifene which could yield the beneficial effects of estrogens and SERMS, while potentially being more tolerable and safer than standard HRT, avoiding the potential deleterious effects of synthetic progestins [24]. The combination of bazedoxifene and CEE has been shown to reduce climacteric symptoms and preserve BMD [45]. TSECs may offer hope to symptomatic women reluctant to take traditional HRT.

\section{Conclusions}

Postmenopausal osteoporosis and related fractures is a serious health threat that can affect nearly half of all white women over the age of 50, and about half of elderly women will have long-term disability, morbidity and death after a fracture. In postmenopausal women who require pharmacologic therapy, available options include ERT/HRT, tibolone, SERMs, and in the near future, TSECS. Despite decades of accumulated evidence demonstrating that HRT is the most effective therapy for the prevention of osteoporosis and related fractures, the United States' Food and Drug Administration (FDA) and the European Medicine Evaluation Agency (EMEA), have recommended not to use HRT as the first-line therapy for prevention of osteoporosis. This advice was based mainly on the presentation and interpretation of the WHI data that were greatly revised by the menopause community $[24,25]$. Years ago we forecasted that the drop in HRT use could have caused an increase in fragility fractures in elderly women [46]. In a recent study, Karim et al. [47] have measured the impact of HRT cessation on the incidence of hip fracture in a large longitudinal study including 80955 postmenopausal women. In this huge cohort of postmenopausal women followed for 6.5 years, HRT discontinuation was associated with a $55 \%$ increase in the risk of hip fracture alone. This study evaluated the incidence of hip fractures and did not analyze the effect of HRT discontinuation on other fractures, such as vertebral or wrist fractures. However, we can assume that the burden of HRT withdrawal, particularly on different skeletal sites, such as vertebral bodies where trabecular bone is prevalent, will be far more substantial. Thus, the Karim study results [48] perhaps even underestimated the overall effect of HRT discontinuation on postmenopausal bone health. This important study cannot be ignored or disregarded by health-care providers and, most importantly, by Regulatory Agencies worldwide [49]. Today there is an urgent need to identify new approaches for long-term osteoporosis prevention. These strategies can include lower HRT doses, SERMs, and TSEC. Unfortunately, data on fracture incidence using lower HRT doses or TSEC are lacking at the moment. Nevertheless, and in the meantime, the current view and recommendations from Regulatory Agencies about HRT need to be revisited and revised without delay.

The use of HRT for osteoporosis prevention is based on biology, epidemiology, animal and preclinical data, observational studies and randomized, clinical trials, and thus bone protection has been included among the benefits of HRT in recent recommendations [24, 25]. In fact, osteoporosis prevention can actually be considered as a major additional effect in perimenopausal women who use HRT for treatment of climacteric symptoms. Appropriate and effective HRT dose and regimen need to be individualized. There is no evidence that alternative treatments to HRT in early postmenopausal period are also beneficial, not only for symptoms but also for osteoporosis prevention. Individual risk-benefit considerations remain subjects for discussions between individual patients and their care-givers. The possibility that LD-HRT or TSEC decrease the fracture risk is not demonstrated but data on bone turnover and density in early postmenopausal women are indicating a strong protective effect and the rationale for future well-conducted prospective studies.

\section{Disclosure}

Authors report no conflicts of interest.

\section{References}

1. Osteoporosis Prevention, Diagnosis and Therapy. NIH Consensus Statement 2000; 17: 1-45

2. Johnell O, Kanis JA. An estimate of the worldwide prevalence and disability associated with osteoporotic fractures. Osteoporos Int 2006; 17 : 1726-1733.

3. Leslie WD, Morin SN. Osteoporosis epidemiology 2013: implications for diagnosis, risk assessment, and treatment. Curr Opin Rheumatol 2014; 26: 440-446.

4. Cummins SR, Melton LJ 3rd. Epidemiology and outcomes of osteoporotic fractures. Lancet 2002; 359: 1761-1767. 
5. Brincat M, Calleja-Agius J, Erel CT, et al; EMAS. EMAS position statement: Bone densitometry screening for osteoporosis. Maturitas 2011; 68: 98-101.

6. Gambacciani M, Spinetti A, de Simone L, et al. The relative contributions of menopause and aging to postmenopausal vertebral osteopenia. J Clin Endocrinol Metab 1993; 77: 1148-1151.

7. Gambacciani M, Ciaponi M. Postmenopausal osteoporosis management. Curr Opin Obstet Gynecol 2000; 12: 189-197.

8. Crandall CJ, Zheng Y, Crawford SL, et al. Presence of vasomotor symptoms is associated with lower bone mineral density: a longitudinal analysis. Menopause 2009; 16: 239-246.

9. Thurston RC, Joffe H. Vasomotor symptoms and menopause: findings from the Study of Women's Health across the Nation. Obstet Gynecol Clin North Am 2011; 38: 489-501.

10. Genazzani AR, Gambacciani M, Schneider HP, et al.; International Menopause Society Expert Workshop. Postmenopausal osteoporosis: therapeutic options. Climateric 2005; 8: 99-109.

11. Reifenstein E, Albright F. The metabolic effects of steroid hormones in osteoporosis. J Clin Invest 1947; 26: 24-56.

12. Gambacciani M, Spinetti A, Taponeco F, et al. Longitudinal evaluation of perimenopausal vertebral bone loss: effects of a low-dose oral contraceptive preparation on bone mineral density and metabolism. Obstet Gynecol 1994; 83: 392-396.

13. Wells G, Tugwell P, Shea B, et al. Meta-analyses of therapies for postmenopausal osteoporosis. V. Meta-analysis of the efficacy of hormone replacement therapy in treating and preventing osteoporosis in postmenopausal women. Endocr Rev 2002; 23: 529-539.

14. Lindsay R, Hart CM, Clark DM. The minimum effective dose of estrogen for prevention of postmenopausal bone loss. Obstet Gynecol 1984; 63: 759-763.

15. Ribot C, Tremollieres F, Pouilles JM. Cyclic Estraderm TTS 50 plus oral progestogen in the prevention of postmenopausal bone loss over 24 months. In: Christiansen C, Overgaard K (eds.). Osteoporosis 1990. Vol 2. Osteopress ApS, Copenhagen 1990; pp. 1979-1984.

16. Effects of hormone replacement therapy on bone mineral density: results from the postmenopausal estrogen/progestin interventions (PEPI) trial. The Writing Group for the PEPI. JAMA 1996; 276: 1389-1396.

17. Lindsay R, Bush TL, Grady D, et al. Therapeutic controversy: estrogen replacement in menopause. J Clin Endocrinol Metab 1996; 81: 3829-3838.

18. Bone HG, Greenspan SL, McKeever C, et al. Alendronate and estrogen effects in postmenopausal women with low bone mineral density. Alendronate/Estrogen Study Group. J Clin Endocrinol Metab 2000; 85: 720-726.

19. Torgerson DJ, Bell-Syer SEM. Hormone replacement therapy and prevention of non-vertebral fractures. A meta-analysis of randomized trials. JAMA 2001; 285: 2891-2897.

20. Schneider DL, Barrett-Connor EL, Morton DJ. Timing of postmenopausal estrogen for optimal bone density. The Rancho Bernado Study. JAMA 1997; 277: 543-547.

21. Rossouw JE, Anderson GL, Prentice RL, et al. Risks and benefits of estrogen plus progestin in healthy postmenopausal women: principal results from the Women's Health Initiative randomized controlled trial. JAMA 2002; 288: 321-333.

22. Chlebowski RT, Hendrix SL, Langer RD, et al. Influence of estrogen plus progestin on breast cancer and mammography in healthy postmenopausal women: the Women's Health Initiative Randomized Trial. JAMA 2003; 289: 3243-3253.

23. Beral V; Million Women Study Collaborators. Breast cancer and hormone replacement therapy in the Million Women Study. Lancet 2003; 362: 419-427.

24. de Villiers TJ, Pines A, Panay N, et al.; International Menopause Society. Updated 2013 International Menopause Society recommendations on menopausal hormone therapy and preventive strategies for midlife health. Climacteric 2013; 16: 316-337.

25. de Villiers TJ, Gass ML, Haines CJ, et al. Global consensus statement on menopausal hormone therapy. Climacteric 2013; 16: 203-204.

26. Yates J, Barrett-Connor E, Barlas S, et al. Rapid loss of hip fracture protection after estrogen cessation: evidence from the National Osteoporosis Risk Assessment. Obstet Gynecol 2004; 103: 440-446.

27. Gambacciani M, Monteleone P, Genazzani AR. Low-dose hormone replacement therapy: effects on bone. Climacteric 2002; 5: 135-139.
28. Gambacciani M, Ciaponi M, Cappagli B, et al. A longitudinal evaluation of the effect of two doses of tibolone on bone density and metabolism in early postmenopausal women. Gynecol Endocrinol 2004; 18: 9-16.

29. Lindsay R, Gallagher C, Kleerekoper M, et al. Effect of lower doses of conjugated equine estrogens with and without medroxyprogesterone acetate on bone in early postmenopausal women. JAMA 2002; 287: 2668-2676.

30. Gambacciani M, Cappagli B, Ciaponi M, et al. Ultra low-dose hormone replacement therapy and bone protection in postmenopausal women. Maturitas 2008; 59: 2-6.

31. Felson DT, Zhang Y. An update on the epidemiology of knee and hip osteoarthritis with a view to prevention. Arthritis Rheum 1998; 41: 1343-1355.

32. Claassen H, Hornberger F, Scholz-Ahrens K, et al. The effect of estrogens and dietary calcium deficiency on the extracellular matrix of articular cartilage in Gottingen miniature pigs. Ann Anat 2002; 184: 141-148.

33. Felson DT, Nevitt MC. The effects of estrogen on osteoarthritis. Curr Opin Rheumatol 1998; 10: 269-272.

34. Gruber HE, Yamaguchi D, Ingram J, et al. Expression and localization of the estrogen receptor-beta in annulus cells of the human intervertebral disc and the mitogenic effect of 17-beta-estradiol in vitro. BMC Musculoskelet Disord 2002; 3: 4

35. Gambacciani M, Pepe A, Cappagli B, et al. The relative contributions of menopause and aging to postmenopausal reduction in intervertebral disk height. Climacteric 2007; 10: 298-305.

36. Muscat Baron Y, Brincat MP, Galea R, et al. Low intervertebral disc height in postmenopausal women with osteoporotic vertebral fractures compared to hormone-treated and untreated postmenopausal women and premenopausal women without fractures. Climacteric 2007; 10: 314-319.

37. Christgau S, Tankó LB, Cloos PA, et al. Suppression of elevated cartilage turnover in postmenopausal women and ovariectomized rats by estrogen and a selective estrogen receptor modulator (SERM). Menopause 2004; 11: 508-518.

38. Gallagher JC, Baylink DJ, Freeman R, et al. Prevention of bone loss with tibolone in postmenopausal women: results of two randomized, double-blind, placebo-controlled, dose-finding studies. J Clin Endocrinol Metab 2001; 86: 4717-4726.

39. Cummings SR, Ettinger B, Delmas PD, et al. The effects of tibolone in older postmenopausal women. N Engl J Med 2008; 359: 697-708.

40. Tamoxifen for early breast cancer: an overview of the randomised trials. Early Breast Cancer Trialists' Collaborative Group. Lancet 1998; 351 1451-1467.

41. Grady D, Ettinger B, Moscarelli E, et al.; Multiple Outcomes of Raloxifene Evaluation Investigators. Safety and adverse effects associated with raloxifene: multiple outcomes of raloxifene evaluation. Obstet Gynecol 2004; 104: 837-844

42. Vogel VG, Costantino JP, Wickerham DL, et al. Effects of tamoxifen vs. raloxifene on the risk of developing invasive breast cancer and other disease outcomes: the NSABP Study of Tamoxifen and Raloxifene (STAR) P-2 trial. JAMA 2006; 295: 2727-2741.

43. Miller PD, Chines AA, Christiansen C, et al. Effects of bazedoxifene on $B M D$ and bone turnover in postmenopausal women: 2-yr results of a randomized, double-blind, placebo- and active-controlled study. J Bone Miner Res 2008; 23: 525-535.

44. Genant HK. Bazedoxifene: a new selective estrogen receptor modulator for postmenopausal osteoporosis. Menopause Int 2011; 17: 44-49.

45. Lobo RA, Pinkerton JV, Gass MLS, et al. Evaluation of bazedoxifene/ conjugated estrogens for the treatment of menopausal symptoms and effects on metabolic bone parameters and overall safety profile. Fertil Steril 2009; 92: 1025-1038

46. Gambacciani M, Ciaponi M, Genazzani AR. The HRT misuse and osteoporosis epidemic: a possible future scenario. Climacteric 2007; 10: 273-275.

47. Karim R, Dell RM, Greene DF, et al. Hip fracture in postmenopausal women after cessation of hormone therapy: results from a prospective study in a large health management organization. Menopause 2011; 18: 1172-1177.

48. Gambacciani M. HRT misuse and the osteoporosis epidemic. Climacteric 2012; 15: 10-11. 